\title{
Influencia de la familiaridad con una ciudad en su conocimiento
}

\author{
Influence of familiarity with a city in its knowledge
}

\author{
Jesús A. Dopico, María Ángeles González y Diego Campos-Juanatey \\ Universidade da Coruña
}

\begin{abstract}
Resumen
Este trabajo tenía por objetivo analizar si el género de los individuos y los años de permanencia en una ciudad influyen de forma significativa en el conocimiento de hitos urbanos. Para el estudio se seleccionó a un grupo de 88 estudiantes del Grado en Administración y Dirección de Empresas de la ciudad de A Coruña. El género de los participantes no influyó de forma significativa sobre el conocimiento de hitos urbanos; sin embargo, las personas que llevaban en la ciudad más de 10 años recordaron más nombres de edificios representativos que aquéllos que llevaban en la ciudad menos de 10 años.

Palabras clave: Hitos urbanos, mapa cognitivo, familiaridad, memoria.
\end{abstract}

\begin{abstract}
This work aimed to analyze whether the gender of the individuals and the years spent in a city significantly influence the knowledge of urban landmarks. A group of 88 students of the Degree in Business Administration from the city of A Coruña was selected for the study. The gender of the participants did not influence the knowledge of urban landmarks; however, people who had been in the city for more than 10 years recalled more names of representative buildings than those who were in the city less than 10 years.

Keywords: Urban landmarks, cognitive map, familiarity, memory.
\end{abstract}

El estudio del mapa cognitivo de la ciudad es objeto de investigación multidisciplinar desde hace décadas, en especial desde el planteamiento original de Lynch y su propuesta de elementos fundamentales en la imagen de la ciudad (Lynch, 1960/2013).

Psicólogos, diseñadores y planificadores ambientales, arquitectos, geógrafos y otros investigadores, están interesados en analizar desde un punto de vista interdisciplinar las influencias del ambiente sobre las personas (García Mira, 1997). Conocer la imagen subjetiva de los habitantes de una ciudad presenta indudables utilidades en cuestiones como la planificación urbana, el diseño de rutas turísticas, la política de movilidad, o las políticas urbanísticas orientadas a dotar a la ciudad de una "buena forma", en el sentido planteado por Lynch (1981), contribuyendo a lo que hoy entendemos como una ciudad sostenible y eficiente en términos socioeconómicos y ambientales (ver Pearce y Fagence, 1996, para una revisión de los planteamientos de Lynch).
De los cinco elementos propuestos por Lynch (sendas, bordes, barrios, nodos e hitos), se ha señalado que los más relevantes para operar en el ambiente serían las sendas, y los hitos o puntos de referencia (Lázaro Ruíz, 2000). Algunas de las variables estudiadas por su influencia en el conocimiento de los puntos de referencia son: las características físicas de esos hitos, la saliencia, o el nivel de contraste con otros lugares del entorno (Appleyard, 1970; Evans, Smith y Pezdek, 1982), y la familiaridad de los individuos con el lugar.

Una medida indirecta de la familiaridad, utilizada en numerosas investigaciones, son los años de permanencia en la ciudad (Carreiras, 1992). Diversos autores han encontrado evidencias de que los años de permanencia en una ciudad tienen un efecto positivo sobre el reconocimiento y recuerdo de hitos urbanos, y sobre la estimación correcta de distancias en la ciudad, entre otros aspectos del conocimiento espacial (Boira, 1992; Campos-Juanatey, Pérez-Fabello y Tarrío, en prensa-a; Evans, Marrero y Butler, 1981; Golledge y Spector, 1978). Centrándonos en los trabajos que han analizado la influencia de la familiaridad en el recuerdo de puntos de referencia, Evans et al. (1981) encontraron que las personas que llevaban más de un año viviendo en la ciudad, recordaban más sendas e hitos que los que llevaban en la ciudad una semana. Campos-Juanatey et al. (en prensa-a) encontraron que las personas que llevaban viviendo en la ciudad de Pontevedra más de un año, tenían un mayor recuerdo de edificios singulares y plazas importantes de la ciudad, que los que llevaban viviendo allí menos de un año.

Otra de las variables personales que ha sido analizada por su posible influencia en la naturaleza y precisión de los mapas cognitivos (en la manera en que se construye el ambiente), es el género de los individuos. En algunas investigaciones se han observado mejores puntuaciones en los hombres en conocimiento espacial, exactitud y extensión de los mapas cognitivos, aunque se ha señalado que las diferencias de género obtenidas en las investigaciones, pueden estar reflejando más las diferencias en la experiencia con el ambiente, que diferentes habilidades cognitivas (Fernández González, 2014; Gifford, 2007).

En esta investigación se pretende averiguar el conocimiento que tienen los alumnos de Administración y Dirección de Empresas (ADE) de la Universidad de A Coruña, de los hitos urbanos (edificios singulares) de la ciudad, y si en este conocimiento tienen influencia 
significativa el género de los individuos y sus años de permanencia en la ciudad.

\section{Método}

\section{Participantes}

En este estudio participó un grupo de 88 estudiantes del Grado en Administración y Dirección de Empresas (ADE) de la Facultad de Economía y Empresa de la Universidad de A Coruña. La distribución por género de los participantes fue la siguiente: 42 hombres, con una media de edad de 21.91 años $(S D=4.31)$, y 46 mujeres, con una media de edad de 21.41 años $(S D=2.63)$.

\section{Material}

Se utilizó una presentación con 20 diapositivas. En cada una de ellas figuraba una fotografía de un hito urbano de la ciudad de A Coruña (edificios públicos y privados, monumentos, iglesias, recintos deportivos, museos, etc.). Los 20 hitos urbanos seleccionados fueron considerados por los experimentadores como los más significativos de la ciudad (ver los nombres de los edificios en la Tabla 1).

\section{Procedimiento}

A los estudiantes, en sus respectivas clases, se les presentaron las 20 diapositivas a un ritmo lento (una diapositiva cada 20 segundos), de forma que cada participante disponía de tiempo suficiente para ver la fotografía de la diapositiva y escribir en un folio el nombre del hito urbano que figuraba en la misma. Los hitos urbanos estaban numerados del 1 al 20 y en la hoja de respuestas los participantes tenían que escribir el nombre del mismo ateniéndose a su numeración en las fotografías proyectadas.

Se computaron los aciertos de cada individuo, considerando acierto cuando éste escribía correctamente el nombre del edificio. Se permitió que la respuesta no estuviese del todo completa (por ejemplo "Colegiata" en lugar de "Colegiata de Santa María" o "Estación de trenes" en lugar de "Estación de San Cristóbal"), o que se indicara uno de los nombres por los que se conoce el edificio (por ejemplo, "Hospital Abente y Lago" o "Antiguo hospital militar"). Se computaron como errores aquéllos en los que el participante confundió el nombre del edificio. No se computaron las respuestas en blanco.

Una vez obtenidos los datos, se dividió a los participantes en función del tiempo de permanencia en la ciudad. En el caso concreto de los estudiantes de ADE de la Universidad de A Coruña se consideró como "baja permanencia" a aquellos que llevaban menos de 10 años viviendo en la ciudad y como "alta permanencia" a los que llevaban residiendo en A Coruña 10 o más años. Esta división se realizó en función de la media de permanencia, que fue de 9.89 años $(S D=9,22)$. La referencia de 10 años a la hora de diferenciar entre alta y baja permanencia en una ciudad ha sido utilizada también por Boira (1992). Del total de participantes, 45 manifestaron que llevaban menos de 10 años de permanencia, y 43, más de 10 años de residencia en la ciudad.

\section{Resultados}

En primer lugar, se deseaba averiguar el nivel de conocimiento de los estudiantes de ADE de los edificios representativos y otros hitos urbanos de la ciudad de A Coruña. Para ello se calculó el porcentaje de recuerdo del nombre de cada uno de los 20 hitos urbanos seleccionados, obteniéndose los resultados que aparecen en la Tabla 1.

Tabla 1.

Porcentaje de veces que se recuerda cada edificio de la ciudad de A Coruña (ordenados de mayor a menor por porcentaje de recuerdo)

\begin{tabular}{lll} 
Orden & Nombre del lugar & Porcentaje \\
\cline { 2 - 3 } 1 & Torre de Hércules & 100 \\
\hline 2 & Estadio de Riazor & 100 \\
3 & Ayuntamiento de A Coruña & 95.45 \\
4 & Marineda City & 94.32 \\
5 & Casa de los peces & 87.5 \\
6 & Estación de trenes San Cristóbal & 82.95 \\
7 & Domus & 79.54 \\
8 & Palacio de Congresos & 76.14 \\
9 & Casa de las Ciencias & 72.73 \\
10 & Castillo de San Antón & 69.32 \\
11 & Palexco & 67.04 \\
12 & Coliseum & 63.64 \\
13 & Teatro Colón & 45.45 \\
14 & Hospital Abente y Lago & 42.04 \\
15 & Fundación Barrié & 34.09 \\
16 & Maestranza Rectorado & 15.91 \\
17 & Kiosco Alfonso & 11.36 \\
18 & Muncyt & 10.23 \\
19 & Colegiata de Santa María & 3.41 \\
20 & Iglesia de Santiago & 2.27 \\
\hline
\end{tabular}

En segundo lugar, se analizó si existían diferencias significativas en el recuerdo de hitos urbanos de la ciudad de A Coruña entre los participantes, en función del género y del tiempo de permanencia en la ciudad. Con esa finalidad se realizó un análisis de varianza (ANOVA) de 2 (altos y bajos en permanencia en la ciudad) x 2 (hombres y mujeres). Como variable dependiente se utilizó el recuerdo correcto del nombre del edificio o hito urbano. Los valores de las medias y las desviaciones típicas del número de aciertos figuran en la Tabla 2.

Tabla 2.

Medias y desviaciones típicas de aciertos y errores en el recuerdo de hitos urbanos de la ciudad de A Coruña

\begin{tabular}{|c|c|c|c|c|c|c|c|c|}
\hline \multirow{4}{*}{$\begin{array}{l}\text { Medidas } \\
\text { Aciertos }\end{array}$} & \multicolumn{4}{|c|}{ Género } & \multicolumn{4}{|c|}{ Permanencia en la ciudad } \\
\hline & \multicolumn{2}{|c|}{ Mujeres } & \multicolumn{2}{|c|}{ Hombres } & \multicolumn{2}{|c|}{ Baja } & \multicolumn{2}{|c|}{ Alta } \\
\hline & $M$ & $S D$ & $M$ & $S D$ & $M$ & $S D$ & $M$ & $S D$ \\
\hline & 11.46 & 3.87 & 11.79 & 3.60 & 9.44 & 3.43 & 13.88 & 2.46 \\
\hline Errores & 2.02 & 1.94 & 1.60 & 1.27 & 1.80 & 1.55 & 1.84 & 1.79 \\
\hline
\end{tabular}

Los resultados del análisis indican que el género de los participantes no influye en el recuerdo de los nombres de los hitos urbanos, $F(1,84)=.435, p=.51$. Sí que existe 
una diferencia significativa en dicho recuerdo, $F(1,84)=$ 47.221, $p<.001$, entre los que llevan residiendo en $\mathrm{A}$ Coruña más de 10 años $(M=13.88 ; S D=2.46)$ y los que llevan en la ciudad menos de 10 años $(M=9.44 ; S D=$ 3.43). La interacción entre las dos variables, permanencia y género, no resultó significativa, $F(1,84)=$ $.375, p=.54$.

Adicionalmente se analizó si el género y los años de permanencia en la ciudad de A Coruña tienen influencia en el número de errores cometidos al recordar los hitos urbanos de la ciudad. Con la finalidad de comprobar dicha hipótesis se realizó un análisis de varianza (ANOVA) de 2 (altos y bajos en permanencia en la ciudad) x 2 (hombres y mujeres), utilizando en esta caso como variable dependiente el número de errores cometidos por los participantes en el recuerdo del nombre de los hitos urbanos mostrados. Las medias y desviaciones típicas del número de errores figuran en la Tabla 2.

En este caso, el análisis de los datos muestra que en el número de errores no influyó de forma significativa el género, $F(1,84)=1.460, p=.23$ ni los años de permanencia en la ciudad, $F(1,84)=.002, p=.96$. Tampoco influyó en el número de errores la interacción entre el género de los participantes y los años de permanencia en la ciudad, $F(1,84)=.313, p=.58$.

\section{Discusión}

Lo primero a destacar de los resultados sobre porcentaje de recuerdo del nombre de hitos urbanos, es que la totalidad de los estudiantes conoce la Torre de Hércules (confirmando el papel central de la Torre de Hércules en la imagen de la ciudad como elemento simbólico y representativo), y el Estadio de Riazor. También el Ayuntamiento de A Coruña y el Centro Comercial Marineda City obtienen porcentajes de recuerdo cercanos al $100 \%$. Estos resultados están en la línea de los obtenidos por González, Dopico y Campos-Juanatey (en prensa), sobre imagen de la ciudad de A Coruña en estudiantes universitarios, aunque en dicho trabajo se utilizó un cuestionario (Boira, 1992) como método de externalización del mapa cognitivo.

Dado que A Coruña cuenta con varios museos científicos, considerados como puntos de referencia e incluidos por este motivo en el estudio, cabe señalar la diferencia entre el grado de recuerdo de los museos científicos municipales, más consolidados (Casa de los Peces, Domus y Casa de las Ciencias), todos con un porcentaje de recuerdo superior al $70 \%$, y el Museo Nacional de Ciencia y Tecnología (Muncyt), de más reciente apertura, que sólo supera ligeramente el 10\% de reconocimiento. Los dos hitos con menor nivel de recuerdo por parte de los estudiantes de ADE (inferior al $4 \%$ ), son los dos edificios religiosos incluidos en el estudio, la Colegiata de Santa María y la Iglesia de Santiago.

El tiempo de permanencia en la ciudad influyó en el recuerdo del nombre de los edificios representativos de la ciudad de A Coruña. Este resultado confirma los obtenidos en otros estudios, que concluyen que una de las variables con mayor influencia en el conocimiento de una ciudad es el tiempo de permanencia en la misma (Campos-Juanatey et al., en prensa-a; Campos-Juanatey, Pérez-Fabello y Tarrío, en prensa-b; Carreiras, 1992; Evans et al., 1981; Golledge y Spector, 1978).

No se encontraron diferencias significativas en el recuerdo de hitos urbanos en función del género, resultado que está en la línea de los obtenidos en otras investigaciones sobre conocimiento de puntos de referencia (Campbell, Hepner y Miller, 2014; Campos-Juanatey et al., en prensa-a; Lázaro Ruiz, 2000). Tampoco en el número de errores cometidos en el recuerdo de hitos urbanos se han encontrado diferencias significativas en función del sexo, ni en función del tiempo de permanencia en la ciudad.

\section{Referencias}

Appleyard, D. (1970). Styles and methods of structuring a city. Environment and Behavior, 2, 100-117.

Boira, J. V. (1992). La ciudad de Valencia y su imagen pública. Valencia: Universidad de Valencia.

Campbell, J. I., Hepner, I. J., y Miller, L. A. (2014). The influence of age and sex on memory for a familiar environment. Journal of Environmental Psychology, 40, $1-8$

Campos-Juanatey, D., Pérez-Fabello, M. J., y Tarrío, S. (en prensa-a). El mapa cognitivo de los estudiantes de Bellas Artes I: El conocimiento de hitos urbanos. Actas del XIII Congreso Internacional Galego-Portugués de Psicopedagoxía. A Coruña.

Campos-Juanatey, D., Pérez-Fabello, M. J., y Tarrío, S. (en prensa-b). El mapa cognitivo de los estudiantes de Bellas Artes II: El conocimiento del centro histórico de Pontevedra. Actas del XIII Congreso Internacional Galego-Portugués de Psicopedagoxía. A Coruña.

Carreiras, M. (1992). Mapas cognitivos y orientación espacial. En J. Mayor y J. L. Pinillos (Eds.), Tratado de psicología general (Vol. 4): Memoria y representación (pp. 375-408). Madrid: Alambra Universidad.

Evans, G. W., Marrero, D. G., y Butler, P. A. (1981). Environmental learning and cognitive mapping. Environment and Behavior, 13, 83-104.

Evans, G. W., Smith, C., y Pezdek, K. (1982). Cognitive maps and urban form. Journal of the American Planning Association, 4, 232-244.

Fernández González, A. (2014). Cognición ambiental de la ciudad: Una aproximación al casco de Ourense. Tesis Doctoral no publicada. Universidade de Santiago de Compostela.

García Mira, R. (1997). La imagen de la ciudad de los barrios de A Coruña. A Coruña: Servicio de Publicaciones de la Universidad de A Coruña.

Golledge, R. G., y Spector, A. N. (1978). Comprehending the urban environment: Theory and practice. Geographical Analysis, 10, 401-426.

González, M. A., Dopico, J. A., y Campos-Juanatey, D. (en prensa). La imagen pública de la ciudad de A Coruña. Actas del XIII Congreso Internacional Galego-Portugués de Psicopedagoxía. A Coruña. 
Gifford, R. (2007). Environmental Psychology. Principles and Practice. Colville, WA: Optimal books.

Lázaro Ruíz, V. (2000). La representación mental del espacio a lo largo de la vida. Zaragoza: Egido Editorial.

Lynch, K. (1960). The image of the city. Cambridge, MA: MIT Press. [Trad. Cast. (2013). La imagen de la ciudad. Barcelona: Gustavo Gili.
Lynch, K. (1981). A theory of good city Form. Cambridge, MA: MIT Press.

Pearce, P. L y Fagence, M. (1996). The legacy of Kevin Lynch. Research Implications. Annals of Tourism Research, 23(3), 576-598. 